\title{
Action Sports Learning Based on Expert Instruction Revised by Wearable Sensor Data
}

\author{
Kazunari Ishida
}

\begin{abstract}
An instruction of expert is one of the important factors for action sports learning of a student and sports education. Video movie recording is also an effective tool to recognize skill level visually. However, the student might misunderstand the instruction because of ambiguity of natural language. Moreover, it is quite difficult to recognize an extremely quick and small action with the video movie. As a practical solution to overcome these problems, this paper reports an example of action sports learning with wearable sensors and time series data analysis. The sensor is composed of three-axis accelerometers, three-axis gyroscopes, three-axis digital compasses, and GPS. To categorize actions based on multiple time series data collected from the sensors, we also developed a classification method of time series data with local cross-correlation function. We apply our method to analyze BMX flatland riding. According to the result, the method can successfully categorize ten types of tricks into appropriate sub categories. We also depict motion timing and sequence among all body parts of an athlete to understand the tricks. Based on the sensor data, the expert can revise the instruction to make the student learn proper way of action sports performance.
\end{abstract}

Keywords - action sports learning, sports education, expert instruction, wearable sensor, time series data, BMX flatland.

\section{INTRODUCTION}

An advice and instruction provided by an expert athlete is one of the important information for students in sports education. Video movie recording of expert athlete and student is also an effective way to find the difference between them visually and to improve student's skill. However, the student might misunderstand the instruction because of ambiguity of natural language. Moreover, it is quite difficult to recognize an extremely quick and small difference of their actions with the video movie. As a practical solution to overcome these problems, this paper reports an example of action sports learning with wearable sensors and time series data analysis method. In recent years, many re-searchers have employed smartphones with micro electro-mechanical systems (MEMS) to collect and analyze this type of human behavior. Due to the widespread use of smartphones in our daily lives, we can use them to record human activities. Ueda et al. [1] used smartphones to collect movement data of human bodies at desks and estimated the status of test subjects as either concentrated on a task or relaxed without any task. However, we need purpose-specific devices to collect sensor data for healthcare

Kazunari Ishida, Hiroshima Institute of Technology and sports applications. Morita et al. [2] developed a measurement device based on a three-axis accelerometer, three-axis gyroscope, and three-axis digital compass coupled with a Bluetooth modem to record body activity (i.e., gait actions, for evaluating the effectiveness of rehabilitation quantitatively). Avci et al. [3] surveyed a wide range of research papers concerning inertial sensing to analyze actions concerning healthcare and sports. Concerning action sports, Harding et. al. [4] used an accelerometer and gyroscope to analyze the variance in the aerial action of snowboarding. Crockett \& Jensen [5] attached a motion capture system to a large motor-driven treadmill to analyze the action dynamics of skateboarding. Ishida [6][7][8] analyzed aerial actions of skateboard, inline skate, and BMX on halfpipe and big air ramp. Campillo et al. [9] mounted measurement equipment on the sprocket of a Bicycle Motocross (BMX) bicycle to analyze the angular velocity and force of pedaling. However, we analyzed balancing and acceleration operation of BMX instead of force of pedaling.

\section{WEARABLE SENSORS AND FLATLAND TRICKS}

Modern BMX flatland tricks are executed on one wheel of BMX instead of both wheels. They can be categorized into five groups, i.e. (1) Scuff, (2) Glide, (3) Spin, (4) Pump, (5) Turbine. Scuff tricks utilize friction of shoe sole to control amount of wheel rotation. Glide tricks obey inertia for uniform linear motion. Spin tricks mean steady turning based on movement of the center of gravity. Pump tricks repeat changing the center of gravity between balance and unbalance to get forward driving force. Turbine tricks repeat the center of gravity to forward and backward alternately to get rotation force. There is big difference between scuff and the other tricks. On one hand, scuff tricks control driving force for rotation of wheel directory. On the other hand, the other trick control the driving force indirectly. We can also categorize the latter tricks into two sub categories. The first category is composed of glide and spin tricks, which do not use any acceleration. The second category is composed of pump and turbine tricks, which utilize acceleration. We discussed four tricks excluding scuff, because tricks without direct drive or friction of wheel are easy to analyse and are difficult to perform. We discuss ten types of BMX flatland tricks, i. e. Peg wheelie glide (Pgw_gd) is moving forward trick with back wheel. Peg wheelie circle glide $\left(\right.$ Pgw_c $_{\text {) }}$ is forward rotation trick. Peg wheelie upside down (Pgw_upsd) is also forward rotation trick although BMX frame was reversed. Time-machine (TMachine) is a highspeed forward spin trick. Mega-spin (Mgspn) is backward spin trick. 
Peg wheelie pump (Pgw_pump). Peg wheelie upside down pump (Pgw_upsd_pump) is forward rotation trick with driving force acceleration. Backyard pump (Byrd_pump) is also forward rotation trick with driving force acceleration although BMX is positioned behind of rider. Peg wheelie turbine (Pgw_turb) is alternately changing driving direction between forward and backward while keeping clockwise rotation direction. Peg wheelie turbine inside (Pgw_turb_in) is almost same with Pgw_turb, a rider uses right or inside axle peg instead of left or outside peg. We put six wearable sensors on a rider's back head, waist, both ankles, and both upper arms. We also put two sensors on a handle bar and frame of BMX.

\section{SIMILARITY AMONG TRICKS}

Cross correlation is one of the suitable notions to calculate similarity between two time-series data. However, an action sports trick contains aperiodic balancing and unbalancing motions. Hence, cross correlation seems to show us low similarity, although two time-series data describe same type of trick, because the correlation focuses on similarity on two entire data series. To avoid the low similarity problem of ordinal cross correlation, we define local cross correlation with time window. However, naïve implementation of the local cross correlation leads to high computation cost. Hence, we developed the incremental calculation method. We defined similarity measure calculated by local cross correlation [10]. This section describes the definition of the local similarity and incremental calculation formulas to reduce calculation time. We calculated local average by formula (1) with respect to a point of time series trajectory represented as $y_{c_{i} \mathrm{i}_{j} j_{\mathrm{p}} \mathrm{p}+\mathrm{t}}$

$$
\mathrm{Y}\left(p_{s}, w_{s} c_{s} i_{s} j\right)=\frac{1}{w} \sum_{t=1}^{W} Y_{c_{j} i j p+t}
$$

sensor axis (j), window size (w), start time of window (p), and time in window $(\mathrm{t})$. The range of $\mathrm{p}$ is $[0,1-\mathrm{w}]$ where 1 is length of time series. We could calculate the local average with formula (1) at the beginning of a time series. The rest of the averages could be calculated incrementally by formula (2).

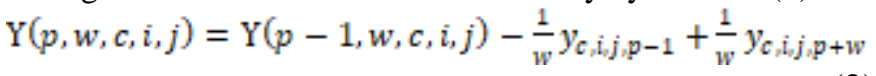

A local variance was calculated by formula (3).

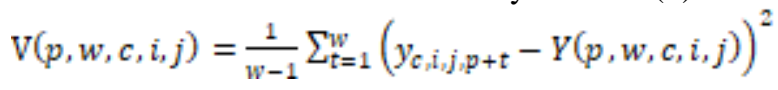

In the same way as the average calculation, we could calculate the rest of the values in each time period with formula (4) and (5) incrementally.

$\mathrm{V}\left(p_{v}, w_{v} c_{v} i_{v} j\right)=\frac{1}{w-1} \mathrm{~V} 2\left(p_{v} w_{v} c_{v} i_{v} j\right)+\frac{w-2}{w-1} Y\left(p, w_{v} c_{v} i_{v} j\right)^{2}$

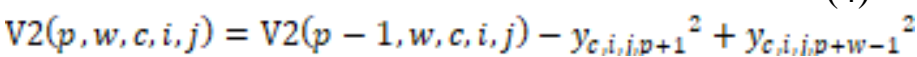

We explained a calculation method of local co-variance between two time series data sets. There were two shift directions between time series data sets $y_{c 1, i j t 1}$ and $y_{d 2 i j t 2}$ to have all local values of co-variance (i.e., $\mathrm{t} 1<\mathrm{t} 2$ and $\mathrm{t} 1>=\mathrm{t} 2$ ). We defined an amount of shift $\mathrm{k}=\mathrm{t} 1 \mathrm{-t} 2$. Common time duration 1 was defined by $\min (\mathrm{n} 1, \mathrm{n} 2-\mathrm{k})$ with negative value of $\mathrm{k}$ or $\min (n 1-k, n 2)$ with positive value of $k$, where $n 1$ and $n 2$ are

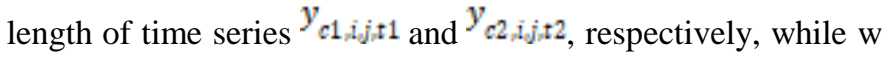
is window size $(\mathrm{w}<=1)$. Local cross-variance between run $\mathrm{c} 1$ and c2 was calculated by formula (6), (7) and (8).

where the parameters are run (c), body part of sensor (i),

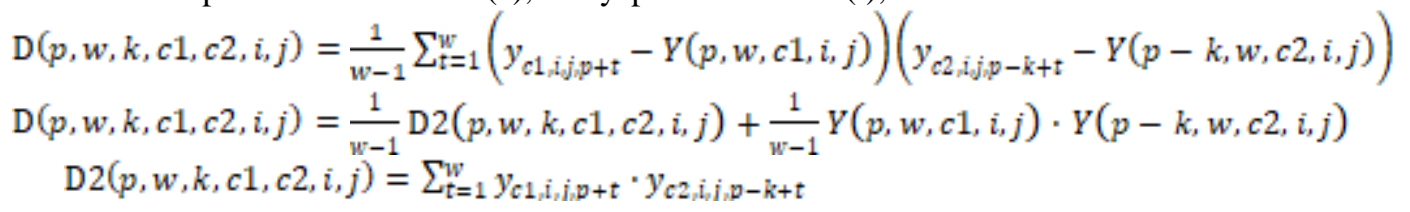

In the same way as average and variance, we can calculate the rest of the values of co-variance at time period $\mathrm{p}$ with (9) incrementally.

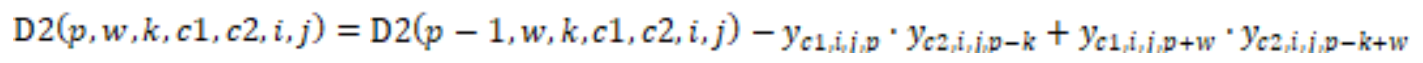

Local cross-correlation coefficient is derived by formula (10), (11), and (12).

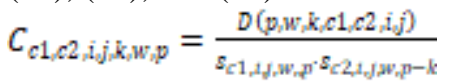

$$
\begin{aligned}
& s_{c 1 \text { ifjwp }}=\sqrt{V\left(p, w_{w} c 1_{s} i_{s} j\right)} \\
& s_{c 2 \text { ijw } p-\mathbb{k}}=\sqrt{V\left(p-k, w_{s} c 2, i, j\right)}
\end{aligned}
$$

We can reduce complexity with the incremental formulation of average, variance, and co-variance. For example, by incremental calculation with window size (w) 200, we could reduce complexity over 200. The window size means 2 seconds when sampling rate is $100 \mathrm{~Hz}$. We put multiple sensors on back of head, waist, right foot, and left foot, which are relatively stable parts of the body. We collected 36 dimensional-data with the four nine-axis sensors. We calculated local cross-correlation coefficient to evaluate similarity between any two runs of data.

\section{A. Cross Correlation}

To understand baseline result of similarity index, we made hierarchical clustering result with ordinal cross correlation (Fig. 1). Fig1 shows us that ordinal cross correlation is not suitable similarity index, because it is not able to categorize ten tricks into proper sub groups. 


\section{B. Local Cross Correlation}

To show the ability of local cross correlation as similarity index, we also made hierarchical clustering result with local cross correlation (Fig. 2). Fig2 shows us that ordinal cross correlation seems to be suitable similarity index, because it shows us proper categorization of the ten tricks.

\section{DISCUSSION}

We discuss characteristics of time series data of each trick to

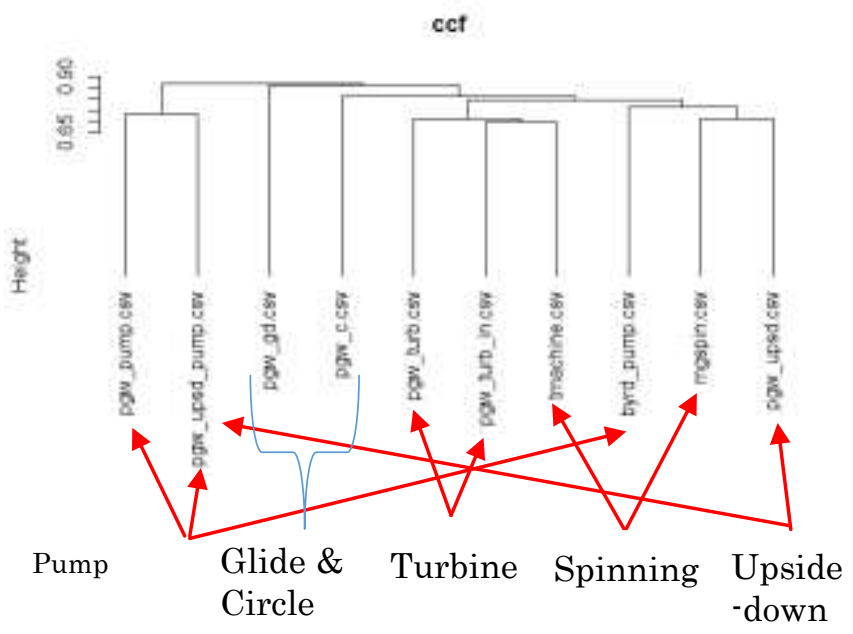

Fig. 1: Clusters with Cross Correlation Similarity.

\section{A. Glide and Spin}

Peg wheelie glide is moving forward trick with back wheel

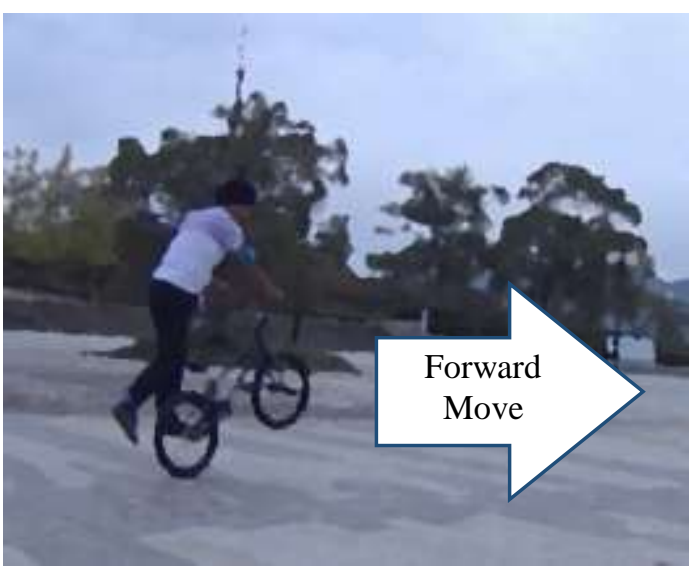

Fig. 3: Peg Wheelie Glide. identify important factors for successful performance. Abbreviations of accelerometer and gyroscope are a and $\mathrm{g}$. Three axes are symbolized as $\mathrm{x}, \mathrm{y}$, and $\mathrm{z}$. We put them on (0) handle bar and (1) frame of BMX. We also put inertial sensors on (2) back-head, (3) waist, (4) right ankle, (5) left ankle, (6) right upper arm, and (7) left upper arm of athlete.

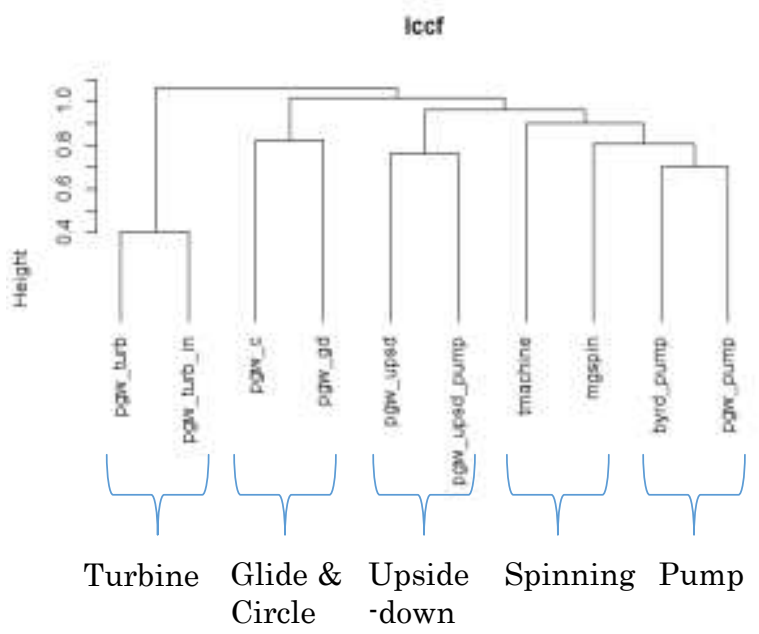

Fig. 2: Clusters with Cross Correlation Similarity.

(Fig. 3). Hence, accelerometer and gyroscope are stable, although there are tiny fluctuations.

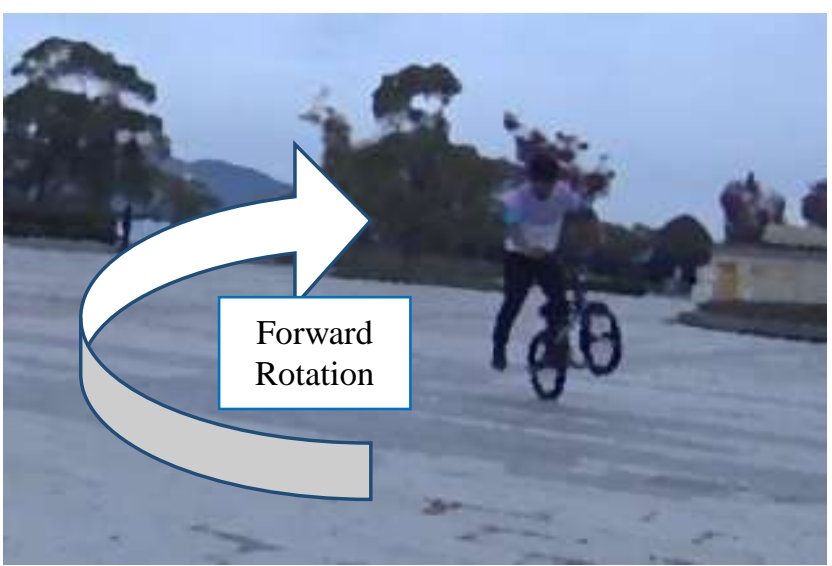

Fig. 4: Peg Wheelie Circle. 


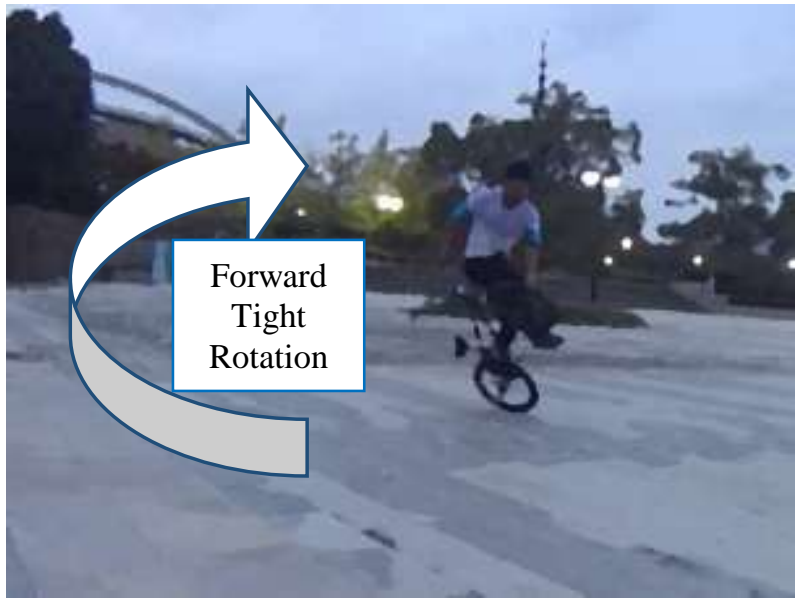

Fig. 5: Time-machine.

Peg wheelie circle glide is forward rotation trick, although it is almost same trick with peg wheelie glide (Fig. 4). Hence, accelerometer and gyroscope are slightly unstable, because the rotation tends to cause unbalance. Time-machine is kind of a highspeed forward spin trick with inward angled frame position to keep the center of gravity (Fig. 5). Mega-spin is backward

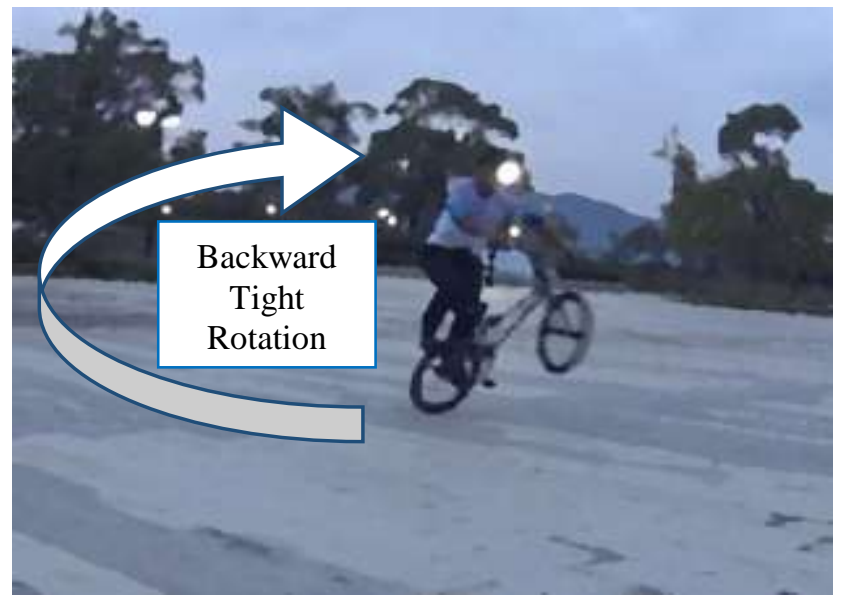

Fig. 6: Mega-spin.

spin trick based on driving back wheel with right foot (Fig. 6).

\section{B. Pump and Turbine}

Peg wheelie pump is forward rotation trick like peg wheelie circle (Fig 7).

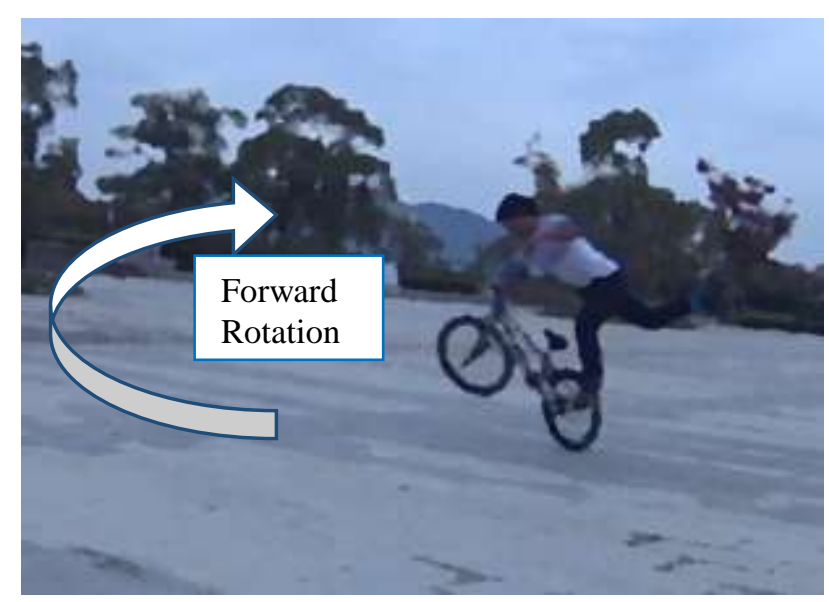

Fig. 7: Peg Wheelie Pump.

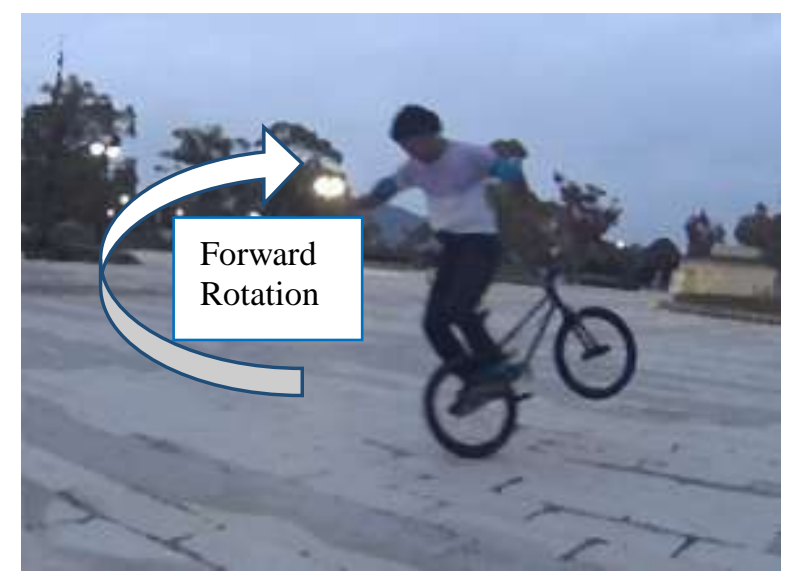

Fig. 9: Backyard pump.

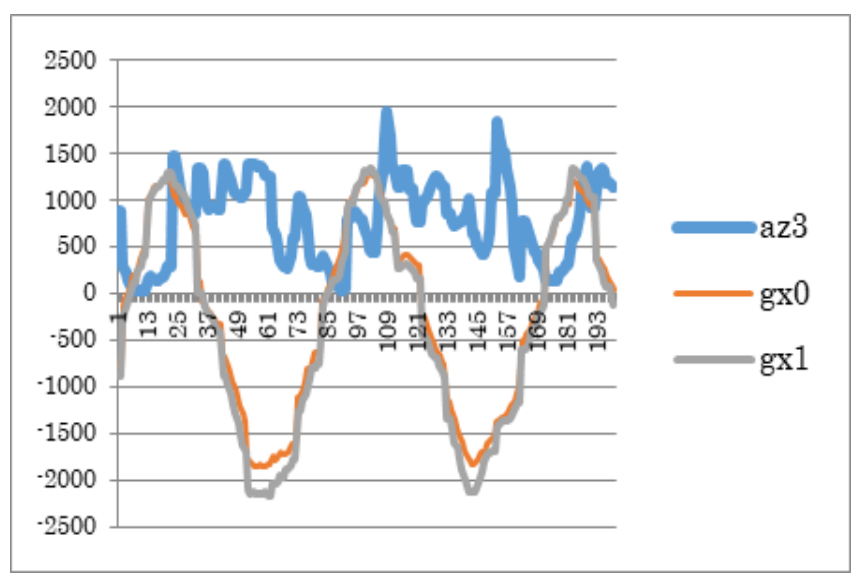

Fig. 8: Peg wheelie pump X-axis gyro (stem, frame).

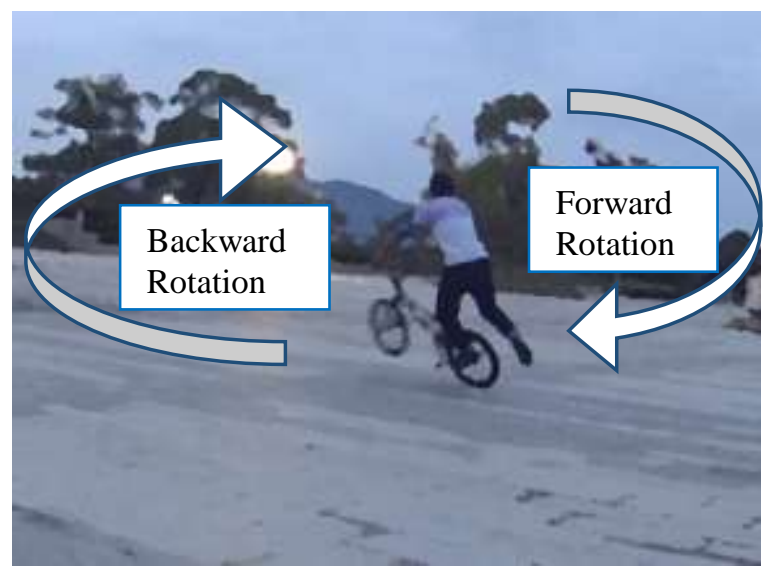

Fig. 10: Peg wheelie turbine. 


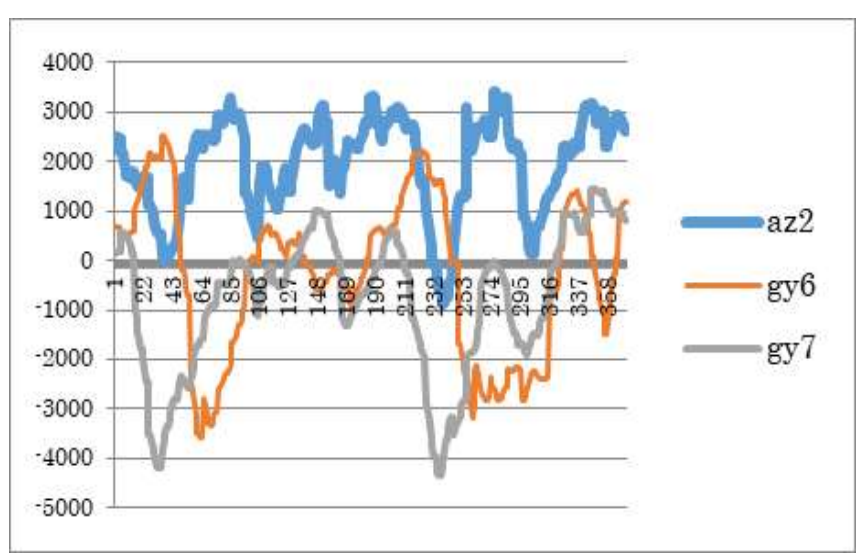

Fig. 11: Peg wheelie turbine upper arms Y-gyro.

Fig. 8 shows us Gravity force on waist accelerometer (gz3) and gyroscope values on handlebar $(\mathrm{gx} 0)$ and frame $(\mathrm{gx} 1)$ of BMX. The pump means intentional balance and unbalance repetition to get driving force. Gravity force on waist (gz3) has zero value when BMX frame become upright position ( $\mathrm{gx} 0$ and gx1 are changing negative to positive values). On the other hand, it has the maximum value when the frame become lean position ( $\mathrm{gx} 0$ and $\mathrm{gx} 1$ are changing positive to negative values).

Backyard pump put BMX behind with both foots on the pegs (Fig. 9). The pump also means intentional balance and unbalance repetition to get driving force.

Peg Wheelie Turbine is alternately changing driving direction between forward and backward while keeping clockwise rotation direction (Fig. 11). The sensor value on back-head vertical accelerometer (az2) depict the characteristics of this trick (Fig. 12). It is composed of alternate short and long term increases. The short increase means backward drive, while the long increase means forward drive.

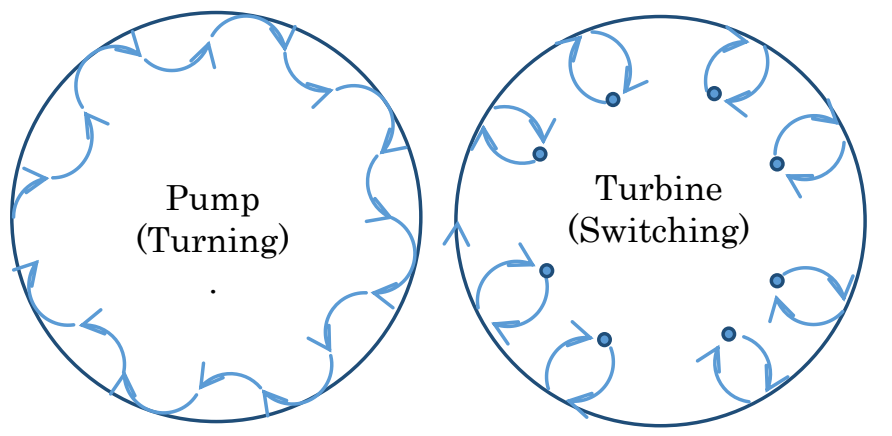

Fig. 13: Difference between Pump and Turbine.

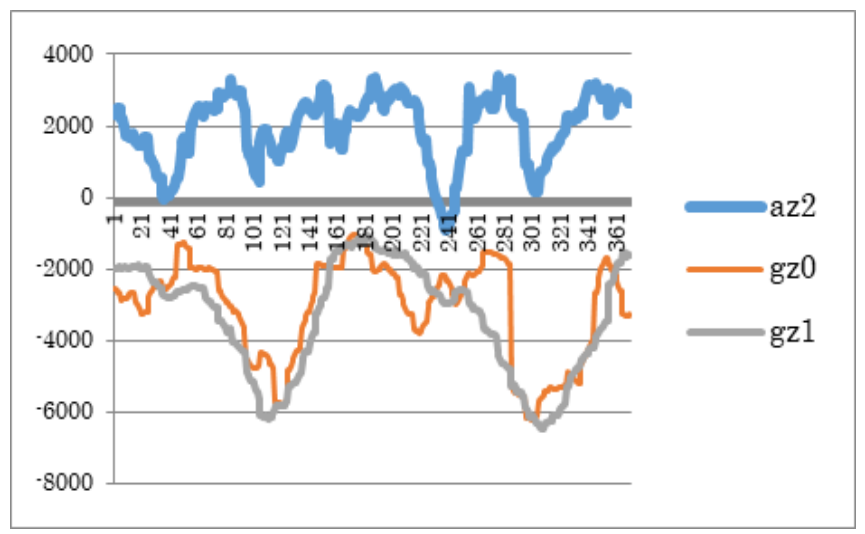

Fig. 12: Peg wheelie turbine stem and frame $\mathrm{Z}$ axis gyro.

To change the driving direction, a rider uses right leg swing. The rider also uses both arms for the direction change. For changing direction from forward to backward, the rider rotate handlebar clockwise with both arm (local maximum gy6 and local minimum gy7). For changing direction from backward to forward, the rider rotate frame clockwise with both arm (local minimum gy6 and gy7).

Pump and Turbine are driving tricks with changing the center of gravity of athlete body and BMX repeatedly. Pump generates driving force with turning, while Turbine generates that with switching (Fig. 13). Both tricks are generating driving force based on the law of conservation of angular momentum.

\section{EXAMPLE OF LEARNING}

We describe learning process of a student, based om the data mentioned in section 4 and an advice provided by an expert.

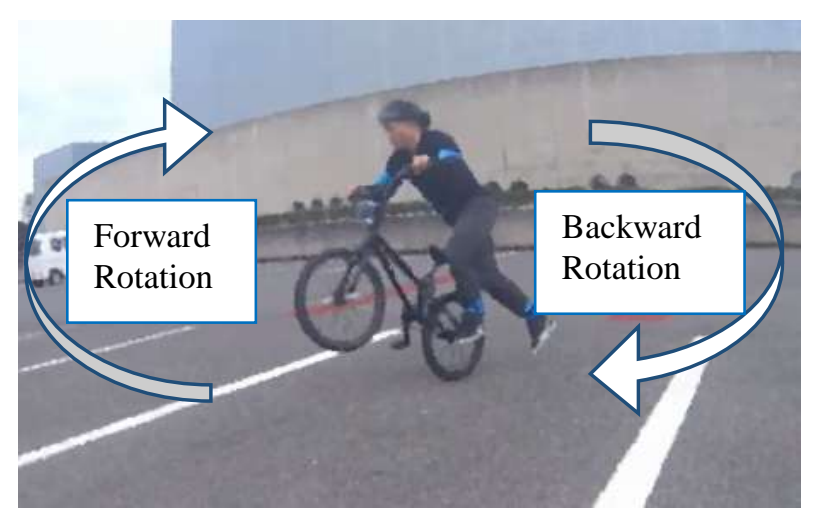

Fig. 14: Peg wheelie turbine. 


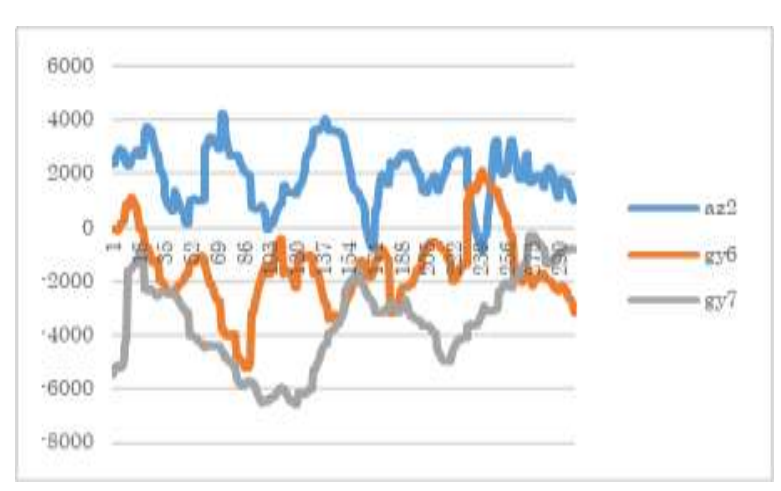

Fig. 15: Peg wheelie turbine Inside Arm Y axis gyro.

The expert taught the student how to do turbine. His advice was that the most important point is control handle by both arms. He told the student like "You have to pull your right or inside arm so that you are able to change driving direction from forward to backward. On the other hand, you must pull your left or outside arm so that you are able to change that from backward to forward. Pulling right and left arms are corresponding to valleys of graph gy6 and gy7 on fig. 11. Based on the advice, the student made attempts of turbine trick. We found that he was able to change driving direction forward to backward, however, he was not able to change that from backward to forward. To solve the learning problem, we analysed expert action data on fig. 11 again. We found that the expert rotates BMX frame clockwise with both arms to change driving direction from backward to forward, although the he did not mention the motion which is one of the most important unconscious technic or skill to do successful turbine (valleys of gz0 and gz1 on fig. 14). After finding the technic, the student tried to employ the technic. His skill was improved so that he can do two rounds turbine trick finally. A round is composed of two direction changes from forward to backward and from backward to forward. Unfortunately, he could not do three round rotation of turbine. To identify wrong motion of the student, we recorded his turbine attempts (Fig. 14). Fig. 15 and 16 depict typical motion of his turbine. According to gy6 in fig. 15, it is not enough to pull handle bar with his right or inside arm. In addition, the second-round rotation of BMX frame is not enough, which is illustrated on small valleys of gz0 and gz1 in fig. 16, although the first-round rotation is enough like expert move. The driving force loss in the second-round rotation seems to be result of wrong motion timing of both arms and right leg. It is future work to improve the student's turbine trick.

\section{CONCLUSION}

This paper identified expert skills concerning BMX flatland tricks. The paper also described an example of leaning process of student to acquire new trick. In the process, we found an unconscious technic or skill of an expert based on sensor data. In future work, we will attempt to develop automatic advice generation system based on difference between expert and student data for supporting sports education.

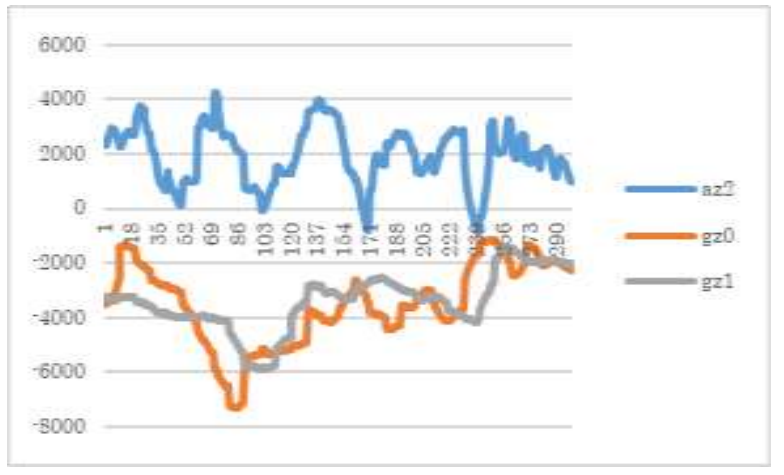

Fig. 16: Peg wheelie turbine stem and frame $\mathrm{Z}$ axis gyro.

\section{ACKNOWLEDGMENT}

We would like to thank Mr. Yoshihiro Nishikawa, who is an international professional BMX flatland rider, because he gives us knowledge and skill of BMX flatland riding. $\mathrm{He}$ is a supported rider of French BMX company St-martin. He won King of Ground competition in 2006, Tokyo. He got second place of FISE competition in 2007, France. He was selected the best 16 riders of Red Bull Circle of Balance in 2007.

\section{REFERENCES}

[1] T. Ueda, H. Sugimura, K. Matsumoto, and M. Isshiki, "Activity recognition of the human from sensor data," The 27th Annual Conference of the Japanese Society for Artificial Intelligence, vol. 86, no. 30, 2013, pp. $1-5$.

[2] Y. Morita, J. Okamoto, K. Kawamura, M. Seki, S. Sessa, M. Zecca, Z. Lin, H. Ishii, S. Takasugi, A. Takanashi, and M. Fujie, "Gait analysis system of presumption of method to estimate right and left separated ground reaction force using acceleration sensor," Welfare, Wellbeing, Life Support (WWLS), 2010, pp. 489-491 (in Japanese).

[3] A. Avci, S. Bosch, M. Marin-Perianu, R. Marin-Perianu, and P. Havinga, "Activity recognition using inertial sensing for healthcare, wellbeing and sports applications: A survey," Architecture of Computing Systems (ARCS), 2010 23rd International Conference, Hannover, Germany, 2010.

[4] J. W. Harding, C. G. Mackintosh, A. G. Hahn, and D. A. James, "Classification of aerial acrobatics," Proceedings of 7th Snowboarding Using Inertial Sensors ISEA CONFERENCE 2008, Biarritz, June 2-6, 2008.

[5] B. A. Crockett and R. L. Jensen, "Kinematic analysis and muscular activity of skateboard propulsion in experienced participants," 25th International Symposium on Biomechanics in Sports, Ouro Preto, Brazil, 2007, p. 602

[6] K. Ishida, "Classification of Turn Maneuvers on Halfpipe with Multiple Sensors," International Journal of Electronics and Electrical Engineering Vol. 4, No. 5, pp. 420-425, 2016.

https://doi.org/10.18178/ijeee.4.5.420-425

[7] K. Ishida, "Action Sports Analysis Based on Local Cross Correlation and Action Measurement Units with GPS timestamp," Third International Workshop on Skill Science, JSAI International Symposia on AI, Kanagawa, November 16, 2016.

[8] K. Ishida, "Analyzing Action Sports Based on Jumping and Aerial Movements with Inertial Sensors and GPS Timestamp," APCST 2017, The 8th Asia-Pacific Congress on Sports Technology, Hilton Hotel, Tel Aviv, Israel, October 15-19, 2017.

[9] P. Campillo, T. Doremus, and J.-M. Hespel, "Pedaling analysis in BMX by telemetric collection of mechanic variables," Brazilian Journal of Biomotricity, vol. 1, no. 2, 2007, pp. 15-27.

[10] K. Ishida, "Application of Computer and Sensor Technology for Analyzing Action Sports," 2018 Asian Conference on Engineering and Natural Sciences (ACENS), International House Osaka, Osaka, Japan, February 6-8, 2018 